\title{
A Short Review on Medication-Overuse Headache
}

\section{Abouch V Krymchantowski*}

Headache Center of Rio, Brazil

\begin{abstract}
Background and objective: Medication-overuse headache $(\mathrm{MOH})$ is a disabling and highly prevalent disease in neurologic clinics. Little evidence is available regarding treatment, but the suspension of overused medications, treatments using multidisciplinary approaches as well as absence of psychiatric comorbidities and overuse of drugs other than opioids are favorable outcome factors.

Methods: We described key patient's characteristics and treatment strategies carried out on consecutive patients with $\mathrm{MOH}$ from a specific tertiary center. The patients were submitted to a comprehensive approach with long-lasting initial consultations. The withdrawal of overused medications was conducted in all patients to whom different drugs for prevention were prescribed as well as the combination of a triptan plus an anti-inflammatory drug (NSAID) in a maximum intake of 2 days/week. Prednisone, as a bridge to detoxification, was given to $67.8 \%$ of the patients during the first 5-7 days.

Results: After two months, $20.1 \%$ of the patients were lost to follow up and the mean headache frequency, among those who adhered, decreased to 10.7 headache days/month. After four and eight months, most of the patients were still under treatment with a mean headache frequency of 7.6 and 8.3 headache days/month. An intention to treat (ITT) analysis was also accomplished. After eight months, relapses or the use of symptomatic medications in 10 or higher days per month was observed in $23.8 \%$ of the patients (ITT $36.2 \%$ ).

Conclusion: The majority of patients with $\mathrm{MOH}$ undergoing comprehensive treatments show marked reduction in headache frequency, return to episodic presentation pattern and reveal acceptable compliance with treatment directives despite previous therapeutic failures. Patient education, thorough evaluation and strict follow up even with patients previously seen as refractory, can lead to optimal clinical outcomes. Controlled studies on different treatment strategies are warranted.
\end{abstract}

Keywords: Medication-overuse headache; Treatment; Withdrawal; Migraine; Chronic migraine

\section{Introduction}

Medication-overuse headache $(\mathrm{MOH})$ is a costly and disabling neurological disease. Its prevalence reaches $3 \%$ of the population, but it may count for up to $70 \%$ of patients from specialized clinics [1]. $\mathrm{MOH}$ is a subgroup of chronic daily headache, which occurs during or it is provoked by overuse of headache symptomatic medications in migraineurs or chronic migraineurs [2,3]. Although it is highly prevalent and promotes considerable burden, little evidence is available regarding treatment, which is mostly based on bias or expert opinion recommendations [4,5]. However, the suspension of overused medications, treatments using multidisciplinary approaches as well as absence of psychiatric comorbidities and overuse of drugs other than opioids are favorable outcome factors [4-7].

The consequences of the absence of robust treatment evidence are that real-world patients are consistently excluded from trials on migraine or chronic migraine with medication-overuse headache, which are mainly performed under the auspices of pharmaceutical industries interested in promoting their current or upcoming products. Therefore, conclusions from these studies usually are not applicable to most of the patients seeking care in specialized clinics $[7,8]$. These subjects are therefore, frequently seen as refractory, difficult or even intractable, but most of the times it was the absence of a previous comprehensive approach the main reason for past treatment failures [5,7,9]. In addition, the theoretical and ineffective assumption that monotherapy is the best treatment option was also responsible for previous frustrating results $[5,7,9]$.

Currently, there is consensus on the need for detoxification with or without the use of bridge medications, which may be carried out on an outpatient basis. Most of the times, it is enough for a successful withdrawal and for a resulting pattern of decreased consumption in overused medications. It also may lead to the reduction of headache frequency as well $[5,7,10,11]$. Additionally, even among patients who succeed in detoxification, there may be a high percentage of those who will relapse within the first year of follow-up [5,6]. Moreover, It is still uncertain whether patients who had daily headache would present a better treatment outcome and experience a higher reduction of headache frequency in comparison to those who had near-daily headache, but few studies suggest that daily headache patients will perform better [12].

\section{Treatment Options}

In this short review, we will focus at describing key patient's characteristics and treatment strategies carried out on consecutive patients with $\mathrm{MOH}$ from a specific tertiary center. It may be not used by everyone, but it does shed light on a path that may guide clinicians in their quest for a better performance of their patients [7,12].

We analyzed recent studies accomplished in sufferers with Medication Overuse Headache ( $\mathrm{MOH})$ according to the International Classification of Headache Disorders (ICHD-3 beta) [13]. The inclusion criteria were 15 or more headache days per month from a primary headache diagnosis of migraine or chronic migraine and overuse of symptomatic medications. The existence of previous attempts of

*Corresponding author: Abouch V Krymchantowski, MD, PhD, Headache Center of Rio, Brazil, Email: abouchkrym@uol.com.br

Received July 03, 2017; Accepted August 18, 2017; Published August 25, 2017

Citation: Krymchantowski AV (2017) A Short Review on Medication-Overuse Headache. Int J Neurorehabilitation 4: 284. doi: 10.4172/2376-0281.1000284

Copyright: ( $) 2017$ Krymchantowski AV. This is an open-access article distributed under the terms of the Creative Commons Attribution License, which permits unrestricted use, distribution, and reproduction in any medium, provided the original author and source are credited. 
treatment or detoxification was not an inclusion limitation as usually happened with most of previous studies [2,6,10,11].

The patients were submitted to a very comprehensive approach with long-lasting initial consultations and written material explaining the nature of the headache and the details of the treatment. Emphatic verbal explanations were presented to all patients, who had to fill out headache calendars on a daily basis. The subsequent visits were scheduled for specific time periods, which allowed a strict follow up process.

The main characteristics of the study population are in Table 1. One hundred sixty eight consecutive patients $(31 \mathrm{M}, 137 \mathrm{~F})$ met the inclusion criteria, but nineteen patients were excluded due the following reasons: presence of personality disorders $(4,4.8 \%)$, refusal to sign the informed consent $(7,4.2 \%)$ and intake of preventive medications during the previous two months $(4,2.4 \%)$. The results from the 149 remaining patients ( 20 Men, 129 Women; $18-65$ years, mean 37.5 ) were studied. Eighty out of 149 patients (53.7\%) reported previous treatment attempts with preventive medications. Among those 80, 52 (65\%) had daily headache and $28(40.6 \%)$ had near-daily headache $(\mathrm{p}<0.0052)$ at baseline. Sixty six reported no improvement and 14 did not adhere to the prescriptions.

The primary headache diagnoses were migraine with aura, without aura or chronic migraine (13) (Table 1). Mean baseline frequency was $24.9 \pm 5.9(16-30)$ headache days/month. Average headache history was $20.9 \pm 11.7$ years (1-37) and the evolution with $\geq 15$ headache days/ month was $4.5 \pm 5.3(0.5-30)$ years. Overuse of rescue medications (RM) was present in all patients, but 59/149 (39.6\%) were overusing more than one pharmacological agent. There were no over users of opioids or barbiturates but 3/149 (2.0\%) patients were overusing benzodiazepines.

The withdrawal of overused medications was conducted in all patients to whom different drugs for prevention were prescribed as well as the combination of a triptan plus an anti-inflammatory drug (NSAID) in a maximum intake of 2 days/week (it was clearly emphasized with the patients). Prednisone, as a bridge to detoxification, was given to 101 patients out of 149 (67.8\%) during the first 5-7 days. The option for using prednisone was based mostly on the expertise of the treating physician, but the higher consumption of overused medications and the presence of comorbidities observed during the initial consultation were

\begin{tabular}{|l|c|}
\hline N & 163 \\
\hline Patients included & 149 \\
\hline Age in years (mean \pm SD) & $37.5 \pm 9$ \\
\hline Gender & Male Female \\
& 20 \\
\hline Daily Headache Patients & $80(53.7 \%)$ \\
\hline Near-Daily Headache Patients & $69(46.3 \%)$ \\
Duration in years of headache (mean \pm SD) & $20.8 \pm 11$ \\
\hline Duration in years of headache>15 days/month (mean) & $0.5-32(4.8)$ \\
\hline Mean days of headache/month & 24.8 \\
\hline Mean days of headache/month (NDH patients) & 18.9 \\
\hline Days of symptomatic medication overuse/month & 22.6 \\
\hline Patients with previous treatment attempts & \\
Daily headache patients & 80 \\
Near-daily headache patients & $52(65 \%)$ \\
Primary headache diagnosis & $28(41 \%)$ \\
\hline Migraine Without aura 131 (72 DH; $59 \mathrm{NDH})$ & \\
\hline Migraine with and without aura 15 (8 DH; 7 NDH) & \\
\hline Chronic Migraine 3 (3 NDH) & \\
\hline Tension-Type Headache 0 & \\
\hline
\end{tabular}

DH: Daily Headache; NDH: Near-Daily Headache

Table 1: Baseline characteristics of the 149 patients studied patients.

\begin{tabular}{|l|c|c|c|}
\hline Preventive treatment choices & $\begin{array}{c}\text { Daily } \\
\text { headaches } \\
\text { (n=80) }\end{array}$ & $\begin{array}{c}\text { Near-daily } \\
\text { headaches } \\
\text { (n=69) }\end{array}$ & $\mathbf{P}^{*}$ \\
\hline Sodium Divalproate & 1 & 11 & 0.0010 \\
\hline Sodium Divalproate+Topiramate & 2 & 6 & 0.0943 \\
\hline Topiramate+Nortriptyline & 6 & 8 & 0.3931 \\
\hline Nortriptyline+Tizanidine+Flunarizine† & 24 & 22 & 0.8040 \\
\hline Nortriptyline+Tizanidine† & 8 & 2 & 0.0841 \\
\hline Sodium Divalproate+(Nortriptyline+Tizanidine)† & 8 & 8 & 0.7540 \\
\hline Sodium Divalproate+Nortriptyline & 4 & 0 & 0.0598 \\
\hline Sodium Divalproate+Candesartane & 3 & 1 & 0.3863 \\
\hline (Nortriptyline+Tizanidine+Flunarizine+Pizotifen)† & 10 & 4 & 0.1620 \\
\hline Topiramate+(Nortriptyline+Tizanidine+Flunarizine)† & 12 & 6 & 0.2390 \\
\hline Topiramate+(Nortriptyline+Tizanidine)† & 2 & 0 & 0.1861 \\
\hline Topiramate+Candesartane & 0 & 1 & 0.2800 \\
\hline
\end{tabular}

*: Chi-square test; $\uparrow$ : Compounded in the same capsule (posology=once a day)

Table 2: Preventive medications prescribed to the included patients in both groups 'daily headaches' and 'near-daily headaches'.

also considered. Unfortunately, no outcome differences between those who used or not prednisone were detected. In addition, no differences were observed among the patients who used it on 5 or on 7 days.

Regarding treatment choices, most of the patients received more than one medication and the single use of sodium divalproate was prescribed to 12 patients (Table 2). Since the choice for medication was not blinded and also based on the treating physician's expertise, the study didn't aim at demonstrating the superiority between the use of specific combinations and the use of more than one preventive agent.

After two months, 30 (20.1\%) patients were lost to follow up and the mean headache frequency, among those who adhered, decreased to 10.7 headache days/month. After four and eight months, respectively 109 and 105 patients were under treatment with a mean headache frequency of 7.6 and 8.3 headache days/month. The intention to treat (ITT) analysis found that the headache frequency after two months, four months, and eight months was, respectively, 13.1; 11.2 and 11.4 headache days/month. After eight months, relapses or the use of symptomatic medications in 10 or higher days per month was observed in 25 patients (23.8\%; ITT $36.2 \%)$.

\section{Discussion}

Although treatment strategies were not studied in a controlled way, the data suggests this is an effective approach in sufferers with an incapacitating condition to which there is no consensus on the best treatment. One might argue on whether counting on recall bias to determine the frequency of headache attacks at baseline is the best option to include and classify the patients, but few studies had a run in period in which the patients just filled out a diary revealing the true headache frequency. Additionally, despite the open methodology used in some of these studies, the findings corroborate the need for a comprehensive approach resulting in interruption of the medication overuse pattern. Moreover, other authors also demonstrated efficient strategies of treatment emphasizing patient's education regarding the biological nature of the headache, withdrawing overused medications abruptly and initiating preventive treatments along with enforcing the correct use of abortive therapy [5].

The effectiveness of the detoxification is also noteworthy. Nearly $80 \%$ of the patients were able to detoxify, which was also carried out in $85.4 \%$ of 376 enrolled patients in other trial [5]. However, there may be discrepancies at the time of deciding on whether preventive treatments 
Citation: Krymchantowski AV (2017) A Short Review on Medication-Overuse Headache. Int J Neurorehabilitation 4: 284. doi: 10.4172/23760281.1000284

are to be initiated. While we decided to use various options with all subjects, others may not do so as with $17.1 \%$ of the patients from other centers [5]. With regard to the discussion on why specific preventive medications were chosen or to claim its effectiveness since it was chosen based in personal experience and not in available evidence, it may be interesting to note that choosing one or more pharmacological agents for the prevention of migraine and $\mathrm{MOH}$ still depends on bias, personal experience or both. In addition, no available evidence is definitive in assuring superiority of drug combinations over monotherapy, but one must consider that studies on that specific objective are purely dependable on commercial interest from pharmaceutical industry and therefore are lacking. Moreover, it is common sense among headache specialists acting in tertiary centers that monotherapy is not the rule.

Another point of discussion, is that regardless of choosing solely evidence-based medicine or, as we have done, deciding for an option that might contemplate the best interest of the patient to improve, we were able to demonstrate a reasonable adherence rate, especially among the daily headache patients ( $88 \%$ after two months and $83.5 \%$ after eight months) although more than half (54\%) demonstrated previous lack of adherence and/or previous treatment failures.

It must be considered that this study had the advantage of a patient population not overusing opioid or barbiturates, which often predicts better treatment outcome figures $[2,6]$. We believe that the results would have being less favorable if these medications were the rule.

On the other hand, although relapse is frequent over time, the majority of the daily headache patients $(67 ; 83.7 \%)$ maintained treatment orientations after 8 months, while only half of the near-daily patients were still under treatment after this time (38;55\%; $\mathrm{p}=0.0001)$. It has to be considered that a higher baseline degree of burden, as with the daily headache sufferers, may result in better compliance with the treatment strategies than with those having headache attacks fewer days a week [14].

The option for a bridge medication during the detoxification period and specifically the option for oral prednisone instead of antidopaminergic agents was also a bias chosen approach and for patients with a higher consumption of acute medications $[2,7,12]$. To do so may lead to less suffering and less use of rescue medication. However, no significant differences were observed regarding compliance and improvement between those who used prednisone and those who not.

\section{Conclusion}

We may conclude that the majority of patients with $\mathrm{MOH}$ undergoing comprehensive treatments show marked reduction in headache frequency, return to episodic presentation pattern and acceptable compliance with treatment directives despite previous therapeutic failures. Patient education, thorough evaluation and strict follow up even with patients previously seen as refractory, can lead to optimal clinical outcomes. Controlled studies on different treatment strategies are warranted.

\section{References}

1. Andrasik F, Grazzi L, Usai S, Kass S, Bussonee G (2010) Disability in chronic migraine with medication overuse: Treatment effects through 5 years. Cephalalgia 30: 610-614.

2. Lipton RB (2015) Risk factors for and management of medication overuse headache. Continuum Minneap Minn 21: 1118-1131.

3. Neves da Silva A, Lake III A (2014) Clinical aspects of medication overuse headaches. Headache 54: 211-217.

4. Russel MB, Lundqvist C (2012) Prevention and management of medication overuse headache. Curr Opin Neurol 25: 290-295.

5. Tassorelli C, Jensen R, Allena M, De Icco R, Sances G, et al. (2014) Consensus protocol for the management of medication-overuse headache: Evaluation in a multicentric, multinational study. Cephalalgia 34: 645-655.

6. Munksgaard SB, Bendtsen L, Jensen R (2012) Treatment-resistant medication overuse headache can be cured. Headache 52: 1120-1129.

7. Krymchantowski AV, Tepper SJ, Jevoux C, Valença M (2017) Medicationoveruse headache: Protocols and outcomes in 149 consecutive patients in a tertiary Brazilian center. Headache 57: 87-96.

8. Evers S, Jensen R (2011) Treatment of medication overuse headache Guideline of the EFNS headache panel. Eur J Neurol 18: 1115-1121.

9. De Felice M, Ossipov MH, Porreca F (2011) Update on medication overuse headache. Curr Pain Headache Rep 15: 79-83.

10. Grande RB, Aaseth K, Benth JS, Lundqvist C, Russel MB (2011) Reduction in medication-overuse headache after short information. The Akershus study of chronic headache. Eur J Neurol 18: 129-137.

11. Rossi P, Faroni JV, Nappi G (2010) Short-term effectiveness of simple advice as a withdrawal strategy in simple and complicated medication overuse headache. Eur J Neurol 18: 396-401.

12. Krymchantowski AV, Tepper SJ, Jevoux C, Valença M (2016) Medicationoveruse headache: Differences between daily and near-daily headache patients. Brain Sci 15: 6.

13. The International Classification of Headache Disorders, $3^{\text {rd }}$ edition (beta version). Cephalalgia 33: 629-808.

14. Hagen K, Albretsen C, Vilming ST, Salvesen R, Gronning M, et al. (2011) A 4-year follow-up of patients with medication-overuse headache previously included in a randomized multicenter study. J Headache Pain 12: 315-322. 